\title{
Cathodoluminescence Microscopy of Self-Organized InGaN Nano-Structures on GaN Pyramids
}

\author{
F. Bertram ${ }^{1}$, S. Metzner ${ }^{1}$, J. Christen $^{1}$, M. Jetter ${ }^{2}$, C. Wächter ${ }^{2}$, and P. Michler ${ }^{2}$ \\ 1 Institute of Experimental Physics, Otto-von-Guericke-University Magdeburg, Germany \\ 2 Institut fuer Halbleiteroptik und Funktionelle Grenzflaechen, Stuttgart University, Germany
}

The luminescence of InGaN nano-structures grown by selective MOVPE is analyzed by spectrally, highly nm-spatially, and ps-time resolved cathodoluminescence (CL) microscopy.

Self organized grown hexagonal GaN pyramids on c-plane sapphire were overgrown by an InGaN single quantum well and capped with a GaN barrier layer. This geometry has strong impact on the InGaN SQW forming quantum dot like potential minima at the pyramid's tips. Intense GaN luminescence can be obtained from both, the pyramids as well as the buffer layer in between. In contrast, the InGaN luminescence is exclusively emitted from the pyramids. The most intense InGaN SQW luminescence originates from the base and the upper part of the pyramids. A striation-like contrast is observed at the lower part of the pyramids, both, in intensity and peak wavelength: higher intensities locally correlate one-by-one with longer peak wavelengths. The overall averaged wavelength is $550 \mathrm{~nm}$. In the upper part of the pyramids two distinctly different wavelengths are emitted: while the center of the facets is dominated by $530 \mathrm{~nm}$ emission a CL peak wavelength of $590 \mathrm{~nm}$ dominates at the edges and at the pyramids tip. This directly visualizes higher indium incorporation and/or a thicker quantum well at the edges and tops of the pyramids, i.e. the self organized formation of quantum wires at the edges and quantum dots at the tips (Fig. 1).

We can directly correlate this real structure with the recombination kinetics of the quantum structures. For a clear understanding of this microscopic kinetics highly spatio-time-resolved techniques are essential. We present results from ps-time- and nm-spatially resolved cathodoluminescence microscopy $(\delta \mathrm{t}<35 \mathrm{ps}, \delta \mathrm{x}<40 \mathrm{~nm})$. The electron beam is extremely fast switched on, stays "on" for a selected time, and is then extremely fast switched off by an electrostatic beam-blanking. This allows the analysis of excitation from thermal equilibrium into true steady state condition and the relaxation back into thermal equilibrium. Consequently, we have chosen adequate long rectangular pulses $(\Delta t=13 \mathrm{~ns})$ for excitation and moderate repetition frequency $(1 \mathrm{MHz})$. The light is detected by a cooled micro-channel plate photomultiplier in single photon-counting system mode in delayed coincidence. The focused electron beam is digitally scanned over the surface $(256 \times 200$ pixels $)$ while CL transients are recorded for a certain dwell time at each pixel. Subsequently, the resulting data set consisting of 51,200 local CL transients i.e. CL(x, $\mathrm{y}, \mathrm{t})$ is stored and evaluated ex situ to produce: local transients, sets of time delayed CL intensity mappings (TDCLI), transient line scans. By use of the box-car method, microscopic lifetime mappings can be generated, directly revealing the nano-scale kinetics in correlation with the morphology. The spatially averaged transient shows strong non-exponential decay (Fig. 2) as a result of the complex dynamics involved. Microscopic local transients taken at the lower part of the pyramids exhibit a very short lifetime down to $\tau_{\text {initial }}=150 \mathrm{ps}$. Upon going up to the top of the pyramid, the life time dramatically increases reaching $\tau_{\text {initial }}>1 \mathrm{~ns}$. Time delayed CL maps TDCLI directly reveal the strong difference of the local lifetime. In particular at the bottom of the pyramids the CL intensity vanishes very fast in less than a few ns after switching off the excitation, whereas at the tip the CL persists even after 34 ns. Normalizing the total lifetime map to the local quantum efficiency yields a microscopic map of the radiative lifetime, which directly correlates the local oscillator strength to the local SQW properties. 

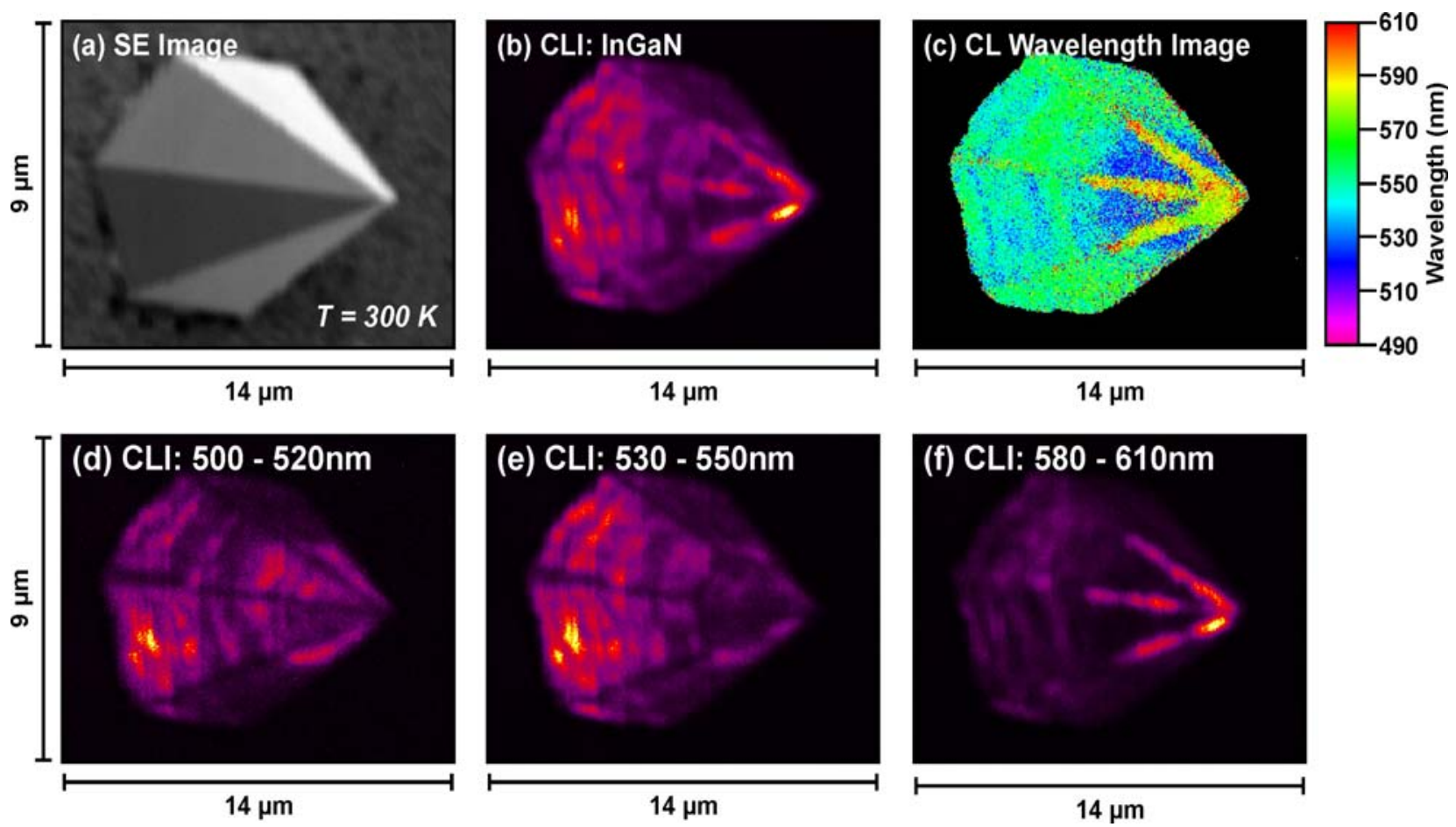

FIG. 1. CL maps of a single pyramid: (a) SE image, (b) panchromatic InGaN CL intensity image (spectrally integrated over the InGaN luminescence), (c) CL peak wavelength image, and (d-f) monochromatic intensity images of different spectral regions of the InGaN luminescence.

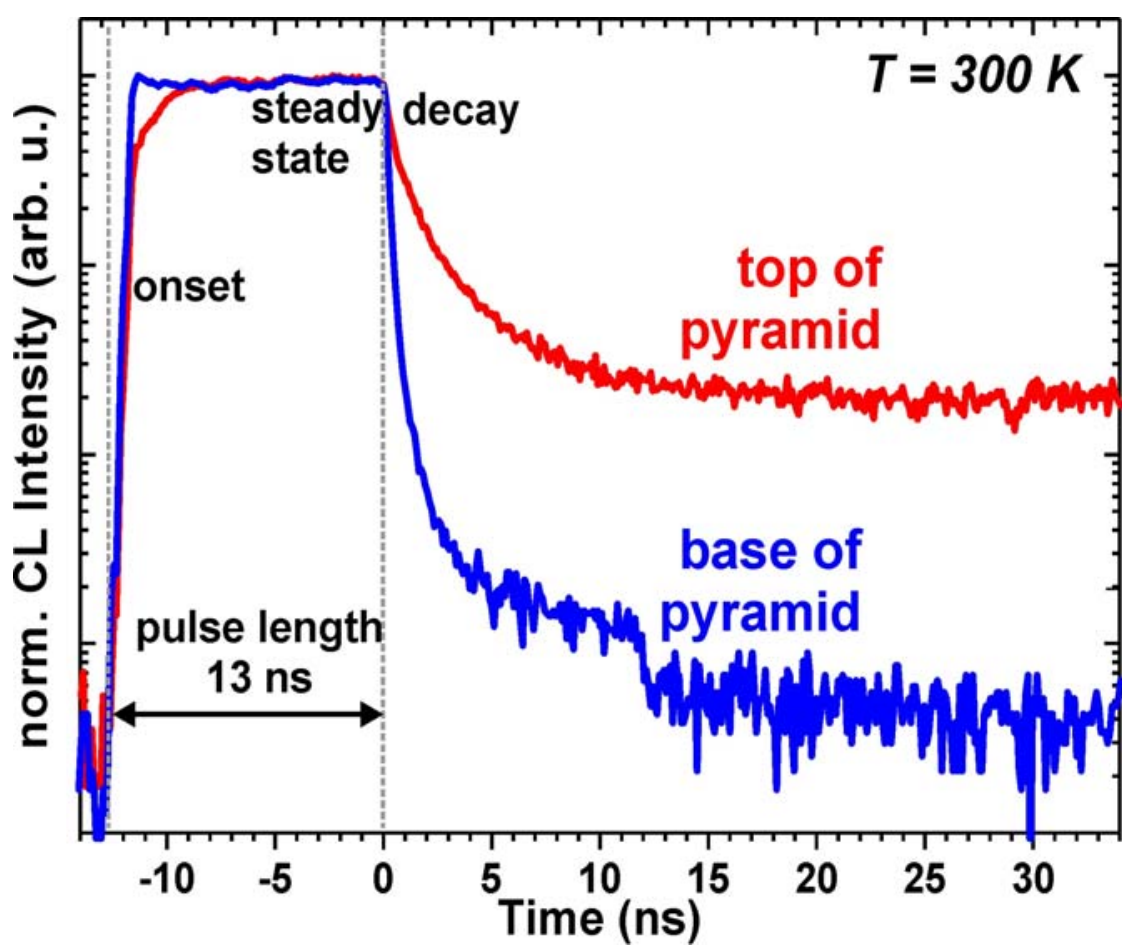

FIG. 2. Local transients taken at the top and the bottom of the pyramid

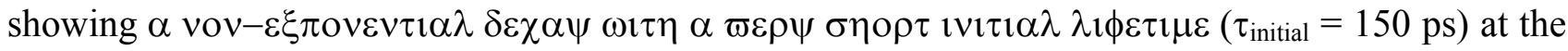
bottom and a slow recombination kinetics at the top of the pyramid $\left(\tau_{\text {initial }}>1 \mathrm{~ns}\right)$. 